\title{
Fast, Adaptive Expectation-Maximization Alignment for Cryo-EM*
}

\author{
Hemant D. Tagare ${ }^{1,2}$, Frederick Sigworth ${ }^{2,3}$, and Andrew Barthel ${ }^{2}$ \\ ${ }^{1}$ Dept. of Diag. Radiology, Yale University, USA \\ hemant.tagare@yale.edu \\ ${ }^{2}$ Department of Biomedical Engineering, Yale University, USA \\ ${ }^{3}$ Department of Physiology, Yale University, USA
}

\begin{abstract}
Cryo-EM is a method for reconstructing 3D structure of proteins without crystallization. The Expectation-Maximization (EM) algorithm is used in the alignment step of Cryo-EM reconstructions. The EM step is often a serious computational bottleneck for 3D reconstructions. This paper proposes a computationally adaptive version of the EM algorithm that speeds up the algorithm by a factor of $20-30$. Experiments with noisy real-world data are included to show that the algorithm achieves this speedup without any significant loss of accuracy. Such speed ups are significant, allowing the reconstruction to converge in cpu-days rather than cpu-months.
\end{abstract}

\section{Introduction}

Cryogenic Electron Microscopy (Cryo-EM) is a relatively new technique for reconstructing the 3D structure of proteins [1]. Cryo-EM images are very noisy (SNR below -10db are common), and high resolution reconstruction is obtained by using many images.

Alignment is an important step in Cryo-EM reconstruction, and the Expectation - Maximization (EM) algorithm is sometimes used for it 234567]. The EM step contains integrals that are computationally very expensive to evaluate and the algorithm can take several cpu-months to converge. There have been two previous approaches to speeding up the EM algorithm. One approach [3|4,5] uses spherical harmonics to simplify some of the integration. This approach is especially promising when the 3 -D structure has icosahedral symmetry. In another, more general approach [7], early iterations of the EM algorithm are examined to isolate a subset of the domain that contributes most to the integral. In subsequent iterations, this subset is held fixed and the integral is evaluated only on it. The problem with this approach is that as iterations proceed, the subset that contributes most to the domain also changes.

In this paper, we present an adaptive EM algorithm where the subset of the domain over which the integral is evaluated changes across iterations. Our

\footnotetext{
* This research was supported by the grant 5R03LM9328-2 from the National Library of Medicine.
} 
algorithm achieves a speedup by a factor that is approximately $20-30$. Thus, cpu-months of computation are reduced to a few cpu-days of computation. In contrast, the algorithm of [7] reports a speedup of a factor of about 7 .

The ultimate proof of any numerical calculation is its ability to give reliable results with real data. We present experimental results to establish that our method gives fast alignment without significant loss of accuracy.

\section{Cryo-EM and the Mixture Model}

Let $S: \mathcal{R}^{3} \rightarrow \mathcal{R}$ be the electron scatter density function of the particle being imaged. Line integrating $S$ along a projection direction $(\theta, \phi)$ (fig 1) gives the projection of $S$ on an image plane perpendicular to $(\theta, \phi)$.

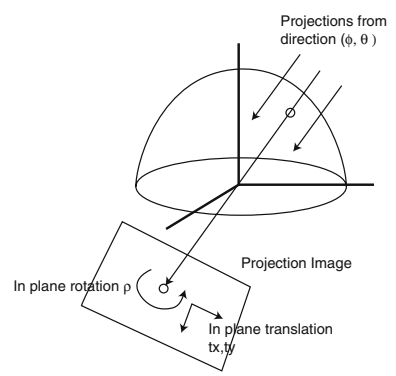

Fig. 1. Cryo-EM Projections

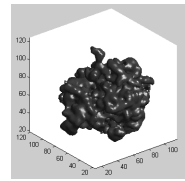

(a) 3D Structure

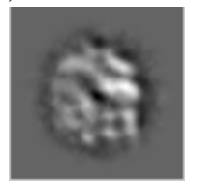

(c) Y-Axis projection (d) Z-axis Projection

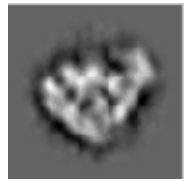

(b) X-axis projection

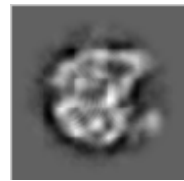

Fig. 2. Ribosome Structure and Projections

An observed Cryo-EM image $I$ is $\mu$ with additive noise and a random inplane rotation and shift. Letting $T_{\tau}$ represent the in-plane image rotation and translation operator, where $\tau=\left(\rho, t_{x}, t_{y}\right)$ is the rotation and translation, the observed image is $I=T_{\tau}(\mu+n)$, or, $T_{-\tau}(I)=\mu+n$, where, $n$ is additive white Gaussian noise. Thus the probability density function (pdf) of observing an image $I$ is

$$
p(I \mid \mu, \sigma)=\int_{\Omega} p_{g}\left(T_{-\tau}(I) \mid \mu, \sigma\right) p(\tau) d \tau,
$$

where, $p(\tau)$ is the density of $\tau, \Omega$ is the support of $p(\tau)$, and

$$
p_{g}\left(T_{-\tau}(I) \mid \mu, \sigma\right)=\frac{1}{(2 \pi)^{P / 2} \sigma} \times \exp \left(-\frac{\left\|T_{-\tau}(I)-\mu\right\|^{2}}{2 \sigma^{2}}\right),
$$

where, \|\| is the usual Euclidean norm, $P$ is the number of pixels in the image, and $\sigma$ is the noise variance of each pixel.

Cryo-EM obtains $N$ images from unknown random projection directions. It is common to model this phenomenon as follows: Let $\theta_{j}, \phi_{j}, j=1, \cdots, M$ be a set 
of fixed projection directions that uniformly sample the unit sphere. Further, let $\mu_{j}$ be the projection of the particle along the $j$ th direction. Then, assuming that any image is a random draw from one of the projections, the pdf of any image $I$ is

$$
p\left(I \mid \mu_{1}, \cdots, \mu_{M}, \sigma, \alpha_{1}, \cdots, \alpha_{M}\right)=\sum_{j=1}^{M} \alpha_{j} p\left(I \mid \mu_{j}, \sigma\right),
$$

where, the mixture coefficients $\alpha_{j}$ are non-negative and sum to 1 , and the component densities $p\left(I \mid \mu_{j}, \sigma\right)$ are given by equation (1) with $\mu=\mu_{j}$.

In this paper, we take the density $p(\tau)$ in equation (11) to be uniform in rotation $\rho$ over $[0,2 \pi)$ and jointly uniform in $t_{x}, t_{y}$ over $[-2,2] \times[-2,2] \mathrm{pixel}^{2}$. Hence $\Omega=[0,2 \pi) \times[-2,2] \times[-2,2]$. These are typical values for Cryo-EM.

Cryo-EM reconstruction has two steps: (1) The estimation of $\mu_{j}$ from the data, and (2) The reconstruction of scattering density $S$ in $3 \mathrm{D}$ from the estimated $\mu_{j}$. The second is the standard 3D tomography step and has many known solutions. We exclusively focus on the first step. In a sense, this step requires that the in-plane rotation and translations of the images be "undone" and the images averaged. Hence, it is called the alignment step.

A small aside to emphasize an important point - typically in equation (2) the value of $\left\|T_{-\tau}(I)-\mu\right\|^{2}$ is several orders of magnitude larger than the value of $\sigma$ (the former is the pixel-wise squared difference summed over the entire image while the latter is the noise variance of a single pixel). Hence in calculating the probability density according to equation (2), small changes in $\left\|T_{-\tau}(I)-\mu\right\|^{2}$ are vastly amplified by the exponentiation.

\section{The EM Algorithm}

The EM algorithm for estimating the parameters of a mixture model is well established [8]. The EM iterations are given below. The superscript $[n-1]$ and $[n]$ refer to the values of the variables in the $n-1$ th and $n$th iteration. The iterations are:

$$
\begin{aligned}
\alpha_{j}^{[n]} & =\frac{1}{N} \sum_{k=1}^{N} \int_{\Omega_{k, j}} p\left(y_{k}=j, \tau_{k} \mid I_{k}, \theta^{[n-1]}\right) d \tau_{k}, \\
\mu_{j}^{[n]} & =\frac{\sum_{k=1}^{N} \int_{\Omega_{k, j}} T_{-\tau_{k}}\left(I_{k}\right) p\left(y_{k}=j, \tau_{k} \mid I_{k}, \theta^{[n-1]}\right) d \tau_{k}}{\sum_{k=1}^{N} \int_{\Omega_{k, j}} p\left(y_{k}=j, \tau_{k} \mid I_{k}, \theta^{[n-1]}\right) d \tau_{k}} \\
\left(\sigma^{2}\right)^{[n]} & =\frac{1}{P} \frac{\sum_{j=1}^{M} \sum_{k=1}^{N} \int_{\Omega_{k, j}} p\left(y_{k}=j, \tau_{k} \mid I_{k}, \theta^{[n-1]}\right)\left\|T_{-\tau_{k}}\left(I_{k}\right)-\mu_{j}^{[n]}\right\|^{2} d \tau_{k}}{\sum_{j=1}^{M} \sum_{k=1}^{N} \int_{\Omega_{k, j}} p\left(y_{k}=j, \tau_{k} \mid I_{k}, \theta^{[n-1]}\right) d \tau_{k}}
\end{aligned}
$$

where, as before, $P$ is the number of pixels in each image. In these formulae, $\Omega_{k, j}=[0,2 \pi) \times[-2,2] \times[-2,2]$ is the domain of integration for $\tau_{k}$ when image 
$I_{k}$ is compared with mean $\mu_{j}$ (all domains $\Omega_{k, j}$ are identical). The function $p\left(y_{k}=j, \tau_{k} \mid I_{k}, \theta^{[n-1]}\right)$ is the latent data probability, and is equal to

$$
p\left(y_{k}=j, \tau_{k} \mid I_{k}, \theta^{[n-1]}\right)=\frac{\alpha_{j}^{[n-1]} p_{g}\left(T_{-\tau_{k}}\left(I_{k}\right) \mid \mu_{j}^{[n-1]}, \sigma^{[n-1]}\right) p\left(\tau_{k}\right)}{\sum_{j=1}^{M} \alpha_{j}^{[n-1]} \int_{\Omega_{i, j}} p_{g}\left(T_{-\tau_{j}}\left(I_{j}\right) \mid \mu_{j}^{[n-1]}, \sigma^{[n-1]}\right) p\left(\tau_{j}\right) d \tau_{j}} .
$$

Usually the integrals in equation (3-6) are numerically evaluated using a grid in each integration domain $\Omega_{k, j}$. The integrand is calculated at each grid vertex and Riemann summed to get the integral. The computationally expensive part of the integral is the calculation of the integrand $\left\|T_{-\tau_{k}}\left(I_{k}\right)-\mu_{j}^{[n]}\right\|^{2}$ at each grid point in $\Omega_{j, k}$. The calculation requires the image to be translated and rotated by the value of $\tau_{k}$ at every grid point. The total number of evaluations of translations and rotations is a good performance measure for the speed of this EM algorithm.

\subsection{Towards an Adaptive EM Algorithm}

We now make an observation which will lead us to the adaptive algorithm: The latent data probabilites in equation (6) are ultimately related to $\| T_{-\tau_{k}}\left(I_{k}\right)-$ $\mu_{j}^{[n-1]} \|$. Now, in practice, it turns out that the function $\left\|T_{-\tau_{k}}\left(I_{k}\right)-\mu_{j}^{[n-1]}\right\|$ varies smoothly with $\tau$ while the latent data probabilities (which are related to the function by exponentiation and normalization) are very spiky. It is the exponentiation step (recall the comment following equation (2)) that converts the smoothly varying function into a spiky latent data probability.

The spiky probabilites are illustrated for the structure of the Ribosome (fig. 2) in figure 3. The "(a)" part of the figure 3 is the z-axis projection of the Ribosome (fig. 2(d)) rotated and corrupted by additive noise (SNR=-17db, typical of CryoEM). Figure $3(\mathrm{~b})$ shows $-\left\|T_{-\tau_{k}}\left(I_{k}\right)-\mu_{j}^{[n-1]}\right\|$ as a function of rotation (the thin curve in the figure). The latent data probability calculated from this is shown in figure 3 (c) (the thin curve again). As argued above, the latent data probability is spiky, while $-\left\|T_{-\tau_{k}}\left(I_{k}\right)-\mu_{j}^{[n-1]}\right\|$ is smoother.

If the domain of support of the spikes in the latent data probability can be found quickly, then numerical integration can be restricted only to those regions and the EM calculations can be done quickly. This is the insight that suggests the adaptive algorithm: First sample $\left\|T_{-\tau_{k}}\left(I_{k}\right)-\mu_{j}^{[n-1]}\right\|$ coarsely, then interpolate and exponentiate it to find which grid points contribute most significantly to the latent data probability. Finally calculate $\mu_{j}^{[n]}$ only using these grid points. This is illustrated in figure 3 as well. The "(b)" part of the figure shows the function sampled coarsely every 15 degrees and interpolated with a cubic B-spline (this is the thick curve). The latent data probabilities calculated from the interpolation are shown in "(c)" also as a thick curve. The latent data probabilities calculated from the interpolation accurately model the support of the exact latent data probabilities.

We can now state the adaptive algorithm. 


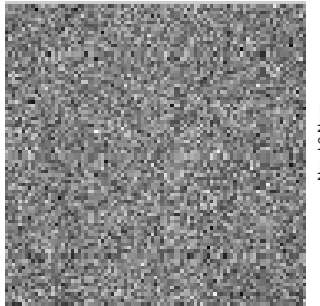

(a) Noisy Image

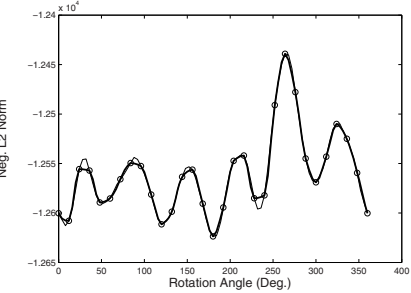

(b) $-\left\|T_{-\tau_{k}}\left(I_{k}\right)-\mu_{j}^{[n-1]}\right\|$

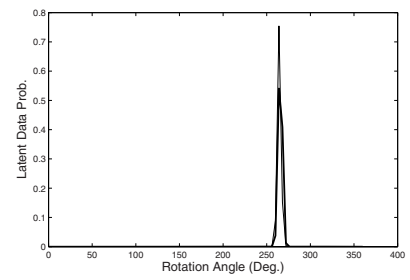

(b) Latent data probability

Fig. 3. Latent Data Probability

\section{The Algorithm}

The adaptive algorithm uses a coarse and a fine grid in each $\Omega_{i, j}$. Each grid partitions the $\Omega_{i, j}$ into cubes. The algorithm first uses the coarse grid to find those cubes which contain spikes of the latent data probability, and then uses numerical integration on the fine grid only in these cubes. The algorithm is as follows:

1. Estimate latent data probability: Calculate $\left\|T_{-\tau_{j}}\left(I_{j}\right)-\mu_{k}^{[n-1]}\right\|^{2}$ at every coarse grid point. Interpolate these values onto the fine grid point using cubic B-splines. Exponentiate the interpolated values, to calculate $p_{g}$ and the latent data probability (eq. (6) ) on the fine grid. At the end of this step we have an estimate of the latent probability at every point in the fine grid.

2. Calculate probability mass: Numerically integrate the latent data probabilities in each coarse grid to get the "probability mass" for every cube in the coarse grid.

3. Identify significant cubes: The next step is to find those coarse cubes which contain most of the "probability mass". These cubes contain the latent data probability spikes. First note that the only domains that contribute to $\mu_{j}^{[n]}$ are $\Omega_{j, k}$ with fixed $j$ (i.e. domains with a different $j$ index do not contribute). Therefore, for the adaptive step, for each $j$, sort the coarse cubes according to increasing latent data probability mass. Starting from the cube with the most probability mass choose as many cubes in reverse order as are needed to get $\zeta$ fraction of the net probability mass ( $\zeta$ in our experiments is 0.99 or greater).

4. Integrate: Calculate the mean according to equation (4) where all integrations are done only on the fine grid in the cubes that are chosen in step 2 . This gives the new estimate $\mu_{j}^{[n]}$.

5. Iterate: Iterate this until the mean estimates $\mu_{j}^{[n]}$ converge.

In this algorithm, the expensive image rotations and translations are done either on the coarse grid (step 1) or on the fine grid in the cubes that survive step 2 . When the latent data probability is spiky, only small percentage of the coarse 
cubes are chosen in step 2 and considerable computational speed up is obtained. Note that speed can be traded in this algorithm for accuracy by setting the parameter $\zeta$ as close as possible to 1 as we want.

\section{Experiments}

We now report experiments with real data using our algorithm. The ribosome structure of figure 2(a) is used in all experiments. The structure has a resolution of $2.82^{\circ} \mathrm{A}$. The structure was projected along 48 directions, a third of which came from a 10 degree cone containing the z-axis, a third around a 10 degree cone containing the y-axis, and a third around a 10 degree cone containing the $\mathrm{x}$-axis. We refer to this by saying that the data came from three classes. Each projection yielded an image of size $92 \times 92$ pixels. White Gaussian noise was added to the data so that the mean S.N.R. in the images was -17 db. Figure 4(a) shows typical images from each class after adding noise. A single noisy image from each class was smoothed and the smoothed images taken to be initial values of the means in the EM iterations (typically initial values come from low resolution reconstructions using other techniques). The EM iterations were carried out with $M=3$ components in two ways: once with the exact EM algorithm (eq. [3] 6 ), and then with the adaptive algorithm of section 4. The adaptive algorithm was deployed using values of $\zeta=0.99,0.999,0.9999$. The convergence criterion for both algorithms was that the r.m.s. difference between successive means was less than $1 \%$ of the r.m.s. values of the means.

The coarse grid sampling was set to 12 degrees for rotation and 2 pixels for $\mathrm{x}$ and y translations. The fine grid sampling was set such that the image transformation between neighboring grid points was at most 1 pixel everywhere in the image. The sampling was $0.5 \mathrm{deg}$ rotation and 0.5 pixel translation. Informal experimentation showed that the results we report below are quite robust with respect to the coarse grid sampling - changing the sampling by $\pm 50 \%$ did not significantly affect the results. The fine grid size is fixed by the size of the image pixel and is algorithm independent.

Figures 4(b-e) show the computed means using the exact EM algorithm and the adaptive EM algorithm. The reconstructions are practically identical. A quantitative analysis of the difference in the reconstructions was obtained by calculating the Fourier Shell Coefficients (FSC). FSCs are the usual method of measuring differences in Cryo-EM [9]. FSCs between two images are calculated in the following way: The Fourier transform of both images is calculated. Then the Fourier plane is binned into $k$ radial "shell" bins from 0 to Nyquist frequency (fig. 4f ). If $D_{k}$ is the domain of the $k^{t h}$ bin in the Fourier space, and $\mathcal{F}(\omega)$ and $\mathcal{G}(\omega)$ are the Fourier Transforms of the two images, then the FSC of the $k^{\text {th }}$ bin is

$$
\frac{\int_{D_{k}} \text { real }\left\{\mathcal{F}^{*}(\omega) \mathcal{G}(\omega)\right\} d \omega}{\sqrt{\int_{D_{k}}\left\{\mathcal{F}^{*}(\omega) \mathcal{F}(\omega)\right\} d \omega} \times \sqrt{\int_{D_{k}}\left\{\mathcal{G}^{*}(\omega) \mathcal{G}(\omega)\right\} d \omega}}
$$

where* denotes complex conjugate. 


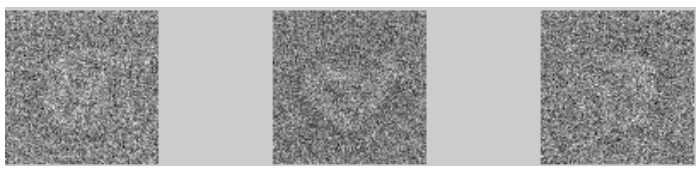

(a) Data images from each class

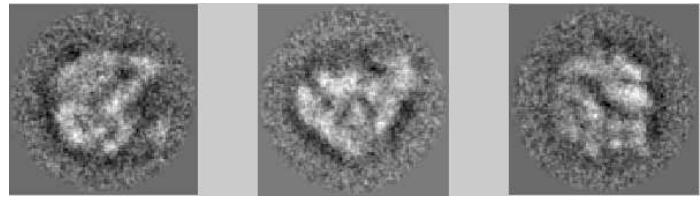

(b) Exact EM estimates of means

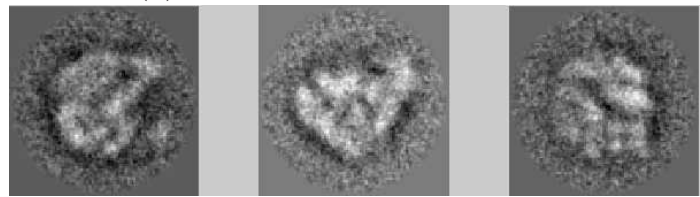

(c) Adaptive EM estimates of mean.

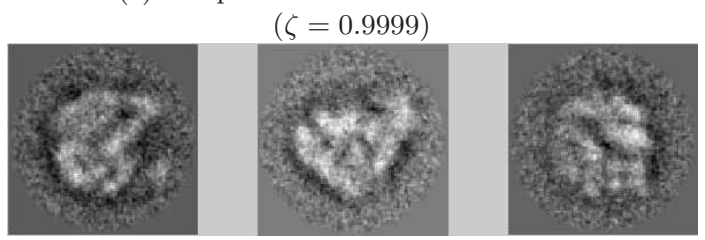

(d) Adaptive EM estimates of mean.

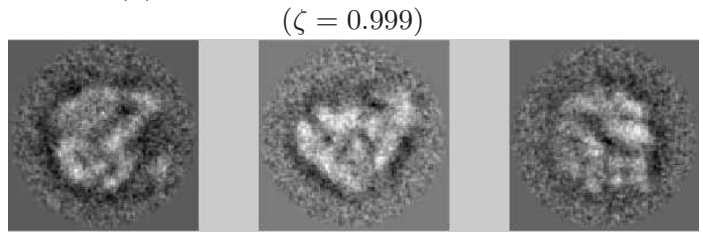

(e) Adaptive EM estimates of mean.

$$
(\zeta=0.99)
$$

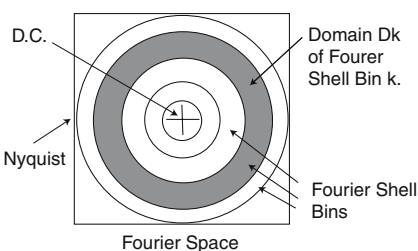

(f) Fourier Shell Bin

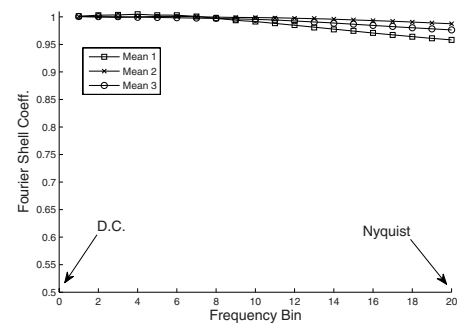

(g) Fourier Shell Correlations $(\zeta=0.9999)$

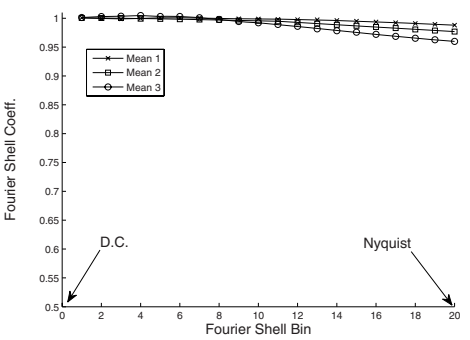

(h) Fourier Shell Correlations $(\zeta=0.999)$

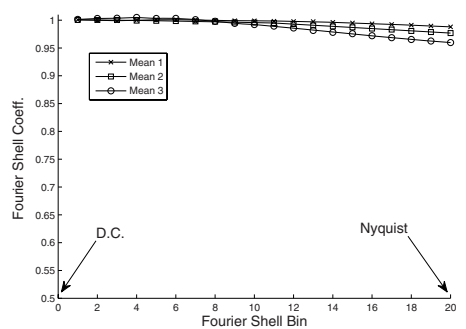

(i) Fourier Shell Correlations $(\zeta=0.99)$

Fig. 4. Complete vs. Adaptive Reconstructions

The FSC take a maximum value of 1 and a minimum value of 0 , with 1 indicating that the Fourier components are identical (modulo scaling) in $D_{k}$. The FSC is calculated for all bins $k$ and plotted as a function of $k$. Figures 4 (g-i) 
shows the FSC for figures 4 (c-e) when compared with figure 4(b). It is quite clear from these values that there is very little difference between the reconstructions.

Finally - and this is the real point of the paper - the speed up of the adaptive algorithm was computed as the ratio of the number of total image transformations required for the exact EM algorithm to the number of image transformations required for the adaptive algorithm. The ratios were $31.2,28.9$, and 25.9 for $\zeta=0.99,0.999,0.9999$ respectively. This shows very clearly that substantial speed ups can be obtained without loss of accuracy using the adaptive algorithm.

\section{Conclusion}

In this paper, we proposed a dual-grid adaptive algorithm that speeds up the EM calculations for the alignment step in Cryo-EM. Experimental evidence shows that the algorithm gives answers which, for all practical purposes, are identical to the exact EM answers. Further, the adaptive algorithm provides a speed up of a factor of 20-30 over the exact EM algorithm making it possible to compute reconstructions in a shorter time or to use larger data sets and thereby reconstructing smaller proteins.

\section{References}

1. Frank, J.: Three Dimensional Electron Microscopy of Macromolecular Assemblies. Oxford University Press, Oxford (2005)

2. Sigworth, F.J.: Classical Detection Theory and The Cryo-EM Particle Selection Problem. J. Mol. Biol. 145(1-2), 111-122 (2004)

3. Zheng, Y., Doerschuk, P.C.: Symmetry-Constrained 3-D Interpolation of Viral XRay Crystallography Data. IEEE Trans. Sig. Proc. 48(1), 214-222 (2000)

4. Yin, Z., Zheng, Y., Doerschuk, P.C., et al.: An ab Initio Algorithm for LowResolution 3-D Reconstructions from Cryoelectron Microscopy Images. J. Struct. Biol. 1(33), 132-142 (2001)

5. Yin, Z., Zheng, Y., Doerschuck, P.C., Natarajan, P., Johnson, J.E.: A Statistical Approach to Processing of Cryo-electron Microscope Images. J. Struct. Biol. 144, 24-50 (2003)

6. Scheres, S.H.W., Valle, M., Nunez, R., Sorzano, C.O.S., Mababini, R., Herman, G.T., Carazo, J.-M.: Maximum-likelihood Multi-reference Refinement for Electron Microscopy Images. J. Mol. Biol. 348, 139-149 (2005)

7. Scheres, S.H.W., Valle, M., Carazo, J.-M.: Fast Maximum-likelihood Refinement of Electron Microscopy Images. Bioinformatics 21(Suppl. 2), 243-244 (2005)

8. McLachlan, G., Peel, D.: Finite Mixture Models. Wiley Interscience, Chichester (2000)

9. van Heel, M., Schatz, M.: Fourier Shell Correlation Threshold Criterion. J. of Struct. Biol. 151(3), 250-262 (2005) 